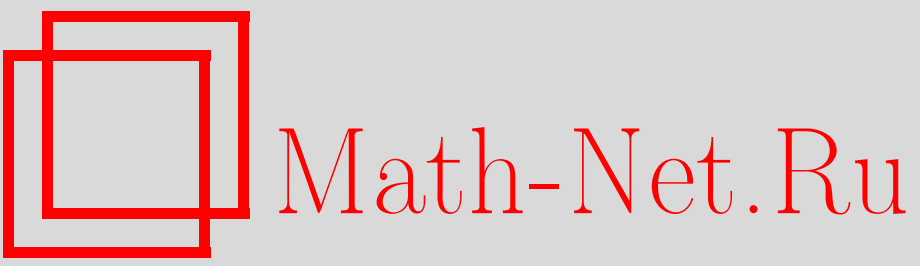

А. В. Тарасов, Свойства систем образующих универсальных алгебр, порождаемых булевыми биюнктивными функциями, Матем. вопр. криптогр., 2012, том 3, выпуск 2, 117-130

DOI: https://doi.org/10.4213/mvk57

Использование Общероссийского математического портала Math-Net.Ru подразумевает, что вы прочитали и согласны с пользовательским соглашением

http://www . mathnet.ru/rus/agreement

Параметры загрузки:

IP : 34.239 .49 .27

26 апреля 2023 г., 17:39:18 


\title{
Свойства систем образующих универсальных алгебр, порождаемых булевыми биюнктивными функциями
}

\author{
A. B. Тарасов \\ Московский государственный технический университет радиотехники, \\ электроники и автоматики, г. Москва \\ Получено 20.V.2011 \\ В работе исследуются два подхода к описанию систем образующих \\ универсальных алгебр, порождаемых булевыми биюнктивными функциями. \\ Основными множествами этих алгебр являются множества выполняющих \\ векторов булевых функций, представимых в виде 2-КНФ; алгебры имеют \\ одну тернарную операцию, определяемую покоординатным применением \\ функции голосования от трех переменных к тройкам двоичных $n$-мерных век- \\ торов. Первый подход состоит в исследовании графов соответствующих \\ 2-КНФ, второй основан на применении задачи о покрытиях множеств.
}

Ключевые слова: 2-КНФ, биюнктивная функция, универсальная алгебра

Properties of generator systems of universal algebras generated by Boolean bijunctive functions

\section{A. V. Tarasov}

Moscow State Technical University of Radio Engineering, Electronics and Automatics, Moscow

\begin{abstract}
Two approaches to the description of generator systems of universal algebras generated by Boolean bijunctive functions are considered. Basic sets of these algebras are sets of satisfying vectors of Boolean functions having 2-CNF form; a ternary operation of these algebras is defined by coordinate-wise application of the voting function to triples of Boolean $n$-dimensional vectors. The first approach is based on the graphs of corresponding 2-CNF, the second is based on the set cover problem.
\end{abstract}

Key words: 2-CNF, bijunctive function, universal algebra

Citation: Mathematical Aspects of Cryptography, 2012, vol. 3, no. 2, pp. 117-130 (Russian). 


\section{Введение}

Пусть $V_{n}$ - множество двоичных векторов длины $n$. Элементы этого множества будем обозначать греческими буквами $\alpha, \beta, \ldots$, а их компоненты через $\alpha(1), \ldots, \alpha(n), \beta(1), \ldots, \beta(n)$ и т. д. Через $0_{n}$ и $1_{n}$ обозначим векторы, состоящие из одних нулей или одних единиц соответственно.

Определим класс биюнктивных функций Ві как класс функций, представимых в виде 2-КНФ:

$$
f\left(x_{1}, \ldots, x_{n}\right) \equiv \wedge_{j=1}^{l} x_{r_{j}}^{b_{j}} \stackrel{t}{\wedge}\left(x_{s_{i 1}} \vee x_{s_{i 2}}^{a_{i}}\right)
$$

пусть $\operatorname{Bi}(n)$ - множество биюнктивных функций, зависящих от $n$ переменных. Через WP обозначим класс слабо положительных функций, т. е. функций, представимых КНФ вида

$$
f\left(x_{1}, \ldots, x_{n}\right) \equiv \hat{j=1}^{l} x_{r_{j}}^{b_{j}} \hat{i=1}_{i=1}^{t}\left(x_{s_{i 1}}^{a_{i}} \vee x_{s_{i 2}} \vee \ldots \vee x_{s_{i_{i}}}\right) .
$$

Для $\alpha, \beta \in V_{n}$ через $\alpha \vee \beta, \alpha \wedge \beta, \alpha \oplus \beta, \bar{\alpha}$ обозначим векторы, получающиеся из $\alpha, \beta$ покоординатным применением соответствующих операций. Например,

$$
\gamma=\alpha \vee \beta \Leftrightarrow \gamma(1)=\alpha(1) \vee \beta(1), \ldots, \gamma(n)=\alpha(n) \vee \beta(n) .
$$

Определим тернарное преобразование $v$ пространства $V_{n}$ равенством

$$
v(\alpha, \beta, \gamma)=(\alpha \wedge \beta) \vee(\alpha \wedge \gamma) \vee(\beta \wedge \gamma), \quad \alpha, \beta, \gamma \in V_{n} .
$$

Универсальную алгебру с основным множеством $V_{n}$ и операцией $v$ обозначим через $\Omega_{n}$.

Через $E_{f}$ обозначим множество выполняющих векторов булевой функции $f\left(x_{1}, \ldots, x_{n}\right)$, т. е. $E_{f}=\left\{\left(x_{1}, \ldots, x_{n}\right): f\left(x_{1}, \ldots, x_{n}\right)=1\right\}$. Введем обозначения: $f_{M}$ - такая булева функция $f\left(x_{1}, \ldots, x_{n}\right)$, что $E_{f_{M}}=M \subseteq V_{n}$, $f_{i_{1} \ldots i_{k}}^{a_{1} \ldots a_{k}}\left(x_{1}, \ldots, x_{n}\right)$ - подфункция функции $f\left(x_{1}, \ldots, x_{n}\right)$, полученная фиксацией переменных $x_{i_{1}}, \ldots, x_{i_{k}}, 1 \leq i_{1}<\ldots<i_{k} \leq n$, значениями $a_{1}, \ldots, a_{k} \in\{0,1\}$,

$$
\begin{gathered}
M_{i j}^{a b}=\left\{\alpha=\left(c_{1}, \ldots, c_{n}\right) \in M: c_{i}=a, c_{j}=b\right\}, \quad 1 \leq i \leq j \leq n, a, b \in\{0,1\}, M \subseteq V_{n}, \\
M \oplus \beta=\{\alpha \oplus \beta: \alpha \in M\}, \quad M \subseteq V_{n}, \beta \in V_{n}, \\
\Lambda=\{(i, j, a, b): 1 \leq i<j \leq n ; a, b \in\{0,1\}\} .
\end{gathered}
$$


Теорема (см. [2]). Булева функиия $f\left(x_{1}, \ldots, x_{n}\right)$ биюнктивна тогда и только тогда, когда для любых трех векторов $\alpha, \beta, \gamma \in E_{f}$ вектор $\delta=v(\alpha, \beta, \gamma)$ является выполняюоиим вектором функиии $f$.

Данная теорема дает возможность при исследовании биюнктивных функций перейти к исследованию универсальных алгебр вида $\Omega_{n}=\left(V_{n}, v\right)$. В [1] изучен ряд свойств алгебр $\Omega_{n}$ и их обобщений: определены их множества эндоморфизмов и элементарных трансляций.

В связи с результатами работ [2-5] возникает вопрос о «минимальных» множествах, порождающих биюнктивные функции с помощью операции $v$. Основная задача, решаемая в настоящей статье, - построение систем образующих подалгебр алгебры $\Omega_{n}$, которые являются множествами выполняющих векторов булевых биюнктивных функций на основе задания их в виде 2-КНФ.

\section{§ 1. Полученные ранее результаты}

Как показано в [3], с помощью подходящего «навешивания отрицаний» на переменные любую биюнктивную функцию можно преобразовать в функцию из класса $\mathrm{Bi} \cap \mathrm{WP}$, представимую в виде

$$
f\left(x_{1}, \ldots, x_{n}\right) \equiv \hat{j=1}_{j=1}^{l} x_{r_{j}}^{b_{j}} \hat{i=1}_{i=1}^{t}\left(x_{s_{i 1}} \vee x_{s_{i 2}}^{a_{i}}\right) .
$$

В дальнейшем будем полагать, что функция $f$ не имеет имплицент, существенно зависящих от одной переменной, т. е. что в (1.1) $l=0$ :

$$
f\left(x_{1}, \ldots, x_{n}\right) \equiv \stackrel{t}{i=1}^{\wedge}\left(x_{s_{i 1}} \vee x_{s_{i 2}}^{a_{i}}\right) .
$$

Функции вида (1.1) и (1.2) лежат в классе $\mathrm{Bi} \cap \mathrm{WP} . \mathrm{B}$ [4] показано, что множество выполняющих векторов таких функций, а следовательно и функций вида (1.2), замкнуто относительно операции покоординатной дизъюнкции и образует нижнюю полурешетку на множестве $V_{n}$ (см. [9]). Для нижних полурешеток определены (см. [5]) понятия минимальных и неразложимых векторов, которыми мы будем далее пользоваться. Через $R_{f}$ обозначим множество неразложимых векторов множества $E_{f}$. Это множество обладает тем свойством, что каждый вектор из $E_{f}$ представим в виде покоординатной дизъюнкции векторов из $R_{f}$.

Теорема 1.1 ([1]). Пусть $f \in \mathrm{WP}$ не имеет имплицент вида $x_{i}^{a}$, $1 \leq i \leq n, \quad a \in\{0,1\}$, u $R_{f} \subset V_{n}-$ множество неразложимых векторов функции $f$. Тогда верно включение $E_{f} \subset\left\langle R_{f} \cup\left\{1_{n}\right\}\right\rangle$, обращающееся в равенство при 
$f \in \mathrm{WP} \cap \mathrm{Bi}$. Если же $f \in \mathrm{WP} \cap \mathrm{Bi} u 1_{n} \in R_{f}$, то для некоторого $i \in 1, \ldots, n$ выполняется условие

$$
f\left(x_{1}, \ldots, x_{n}\right) \leq \underset{j \neq i}{\wedge}\left(\bar{x}_{i} \vee x_{j}\right) .
$$

Определим множества $X=\left\{x_{1}, \ldots, x_{n}\right\}, \bar{X}=\left\{\bar{x}_{1}, \ldots, \bar{x}_{n}\right\}$ и для 2-КНФ вида (0.1) определим ориентированный граф $G=(V, \varepsilon)$ :

$$
V=X \cup \bar{X}, \quad \mathcal{E}=\left\{\left(x_{s_{i 1}}^{a_{i 1} \oplus 1}, x_{s_{i 2}}^{a_{i 2}}\right),\left(x_{s_{i 2}}^{a_{i 2} \oplus 1}, x_{s_{i 1}}^{a_{i 1}}\right), \quad i=1, \ldots, t\right\} .
$$

Напомним, что наличие аффинных имплицент функции $f$ однозначно определяется наличием сильно связных компонент в графе $G$ (см. [3]).

$\mathrm{B}$ соответствии с [6] класс $\mathrm{WP} \cap \mathrm{WN}$ является подклассом класса $\mathrm{Bi}$, содержащим функции вида

$$
f\left(x_{1}, \ldots, x_{n}\right) \equiv\left(\sum_{i=1}^{t_{1}} x_{s_{i}}^{a_{i}}\right) \wedge\left(\underset{i=t_{1}+1}{\stackrel{t}{\&}}\left(\bar{x}_{s_{i 1}} \vee x_{s_{i 2}}\right)\right) .
$$

Множество выполняющих векторов $E_{f}$ такой функции образует дистрибутивную решетку. Для такой решетки можно сравнительно легко описать как множества неразложимых векторов $R_{f}$, так и множества конеразложимых векторов со- $R_{f}([6,9])$. Наиболее наглядно эти множества описываются, когда функция $f$ не имеет аффинных имплицент. Тогда ее можно записать в виде

$$
f\left(x_{1}, \ldots, x_{n}\right) \equiv \stackrel{t}{i=1}_{i=1}\left(\bar{x}_{s_{i 1}} \vee x_{s_{i 2}}\right) .
$$

В графе 2-КНФ такой функции нет дуг, соединяющих вершины множеств $X$ и $\bar{X}$, и можно рассматривать его подграф $G_{1}$, порожденный множеством вершин $X: G_{1}=G(f)(X)$. Множество дуг такого графа обозначим через $\varepsilon_{1}$.

Утверждение 1.2 ([1]). Пусть $f \in \mathrm{WP} \cap \mathrm{WN}$ и граф $G_{1}$ не илеет сильно связных компонент. Тогда множество $R_{f}$ состоит из $n+1$ векторов $R_{f}=\left\{\alpha_{0}, \alpha_{1}, \ldots, \alpha_{n}\right\}$, где $\alpha_{0}=0_{n}$, а векторы $\alpha_{i}, i=1, \ldots, n$, построены по принuипу $\alpha_{i}(i)=1$ и $\alpha_{i}(j)=1$ для всех таких $j$, что вершина $x_{j}$ достижима из вершины $x_{i}$, т.е. в графе $G_{1}$ существует ориентированный путь из вершины $x_{i}$ в вершину $x_{j}$, и $\alpha_{i}(j)=0-$ в противном случае.

Утверждение 1.3 ([1]). Пусть $f \in \mathrm{WP} \cap \mathrm{WN}$ и граф $G_{1}$ не имеет сильно связных компонент. Положим

$$
g\left(x_{1}, \ldots, x_{n}\right)=f\left(x_{1} \oplus 1, \ldots, x_{n} \oplus 1\right) \in \mathrm{WP} \cap \mathrm{WN} .
$$


Тогда множества неразложимых и конеразложимых векторов функиий $f$ ug связаны соотношениями

$$
R_{f}=\left(\operatorname{co}-R_{g}\right) \oplus 1_{n}, \operatorname{co}-R_{f}=R_{g} \oplus 1_{n} .
$$

Пусть $f \in W P \cap W N$, граф $G_{1}=G(f)(X)$ минимальной 2-КНФ не имеет сильно связных компонент. Обозначим через $\operatorname{deg}^{+}\left(x_{i}\right)$ и $\operatorname{deg}^{-}\left(x_{i}\right)$ соответственно полустепени захода и исхода вершины $x_{i}$, и пусть $R_{f}=\left\{\alpha_{0}, \alpha_{1}, \ldots, \alpha_{n}\right\}$ определено как в лемме 1 . В силу того, что граф $G_{1}$ не имеет сильно связных компонент, для каждой его вершины $x_{i}, \quad i=1, \ldots, n$, можно рассмотреть подграф $T\left(x_{i}\right)$, построенный на вершинах, достижимых из $x_{i}$. Данный граф, очевидно, имеет единственную начальную вершину $x_{i}$ и не имеет контуров. Для дальнейшего изложения важное значение имеет следующая теорема.

Теорема 1.4 ([1]). Пусть в определенных выше условиях для некоторой вериины $x_{i}, \quad i \in\{1,2, \ldots, n\}$, графа $G_{1}$ полустепень захода удовлетворяет условию $\operatorname{deg}^{+}\left(x_{i}\right) \geq 2$. Тогда, если в графе существуют такие вериины $x_{j}, x_{k}$, что $\left(x_{j}, x_{i}\right),\left(x_{k}, x_{i}\right) \in \varepsilon_{1}$ и при этом выполнено условие $T\left(x_{j}\right) \cap T\left(x_{k}\right)=T\left(x_{i}\right)$, $m o\left\langle R_{f}\right\rangle=\left\langle R_{f} \backslash\left\{\alpha_{i}\right\}\right\rangle$.

\section{§ 2. Системы образующих алгебр и графы 2-КНФ}

Определение 2.1. Вектор $\delta \in E_{f}$, для которого существуют отличные от него векторы $\alpha, \beta, \gamma \in E_{f}$, удовлетворяющие условию $\delta=v(\alpha, \beta, \gamma)$ (см.(0.3)), называется производнылм вектором функции $f$.

Определение 2.2. Булевы функции $f\left(x_{1}, \ldots, x_{n}\right)$ и $g\left(x_{1}, \ldots, x_{n}\right)$ называются подобными, если найдутся такие подстановка $\sigma \in S_{n}$ и вектор $\alpha=\left(a_{1}, \ldots, a_{n}\right) \in V_{n}$, что

$$
f\left(x_{1}, \ldots, x_{n}\right) \equiv g\left(x_{\sigma(1)} \oplus a_{1}, \ldots, x_{\sigma(n)} \oplus a_{n}\right) .
$$

Следующая теорема дает полное описание биюнктивных функций, не имеющих производных векторов.

Теорема 2.3. Пусть функция $f\left(x_{1}, \ldots, x_{n}\right) \in \operatorname{Bi}(n)$ не имеет аффинных имплицент и существенно зависит от всех переменных. Функция $f$ не имеет производных векторов тогда и только тогда, когда она подобна функичи

$$
g\left(x_{1}, \ldots, x_{n}\right)=\left(\bar{x}_{1} \vee x_{2}\right)\left(\bar{x}_{2} \vee x_{3}\right) \ldots\left(\bar{x}_{n-1} \vee x_{n}\right) .
$$

Доказательство. Утверждение теоремы эквивалентно утверждению о том, что граф минимальной 2-КНФ функции $f$ является ориентированным путем. По условию теоремы в данном графе нет циклов. 
Докажем теорему в прямую сторону от противного. Пусть в графе минимальной 2-КНФ есть вершина с полустепенью захода (или исхода) не меньше 2. Без ограничения общности считаем, что это вершина $x_{3}$. Предположим, что $x_{1}$ и $x_{2}$ - вершины графа, из которых выходят дуги в вершину $x_{3}$. В силу того, что рассматривается граф минимальной 2-КНФ, возможен только один вариант, а именно когда вершины $x_{1}$ и $x_{2}$ взаимно не достижимы. Тогда простыми имплицентами функции $f$ являются функции $\bar{x}_{1} \vee x_{3}$ и $\bar{x}_{2} \vee x_{3}$. Поэтому в $E_{f}$ найдутся такие векторы $\alpha=(0,0,0, \ldots), \quad \beta=(1,0,1, \ldots), \gamma=(0,1,1, \ldots)$, что $\delta=v(\alpha, \beta, \gamma)=(0,0,1, \ldots) \in E_{f}$. Это означает, что функция $f$ подобна произведению функций вида

$$
h_{k}\left(x_{1}, \ldots, x_{k}\right)=\left(\bar{x}_{1} \vee x_{2}\right)\left(\bar{x}_{2} \vee x_{3}\right) \ldots\left(\bar{x}_{k-1} \vee x_{k}\right),
$$

зависящих от непересекающихся наборов переменных, т. е.

$$
f\left(x_{1}, \ldots, x_{n}\right)=h_{k_{1}}\left(x_{s_{1}^{(1)}}, \ldots, x_{s_{k_{1}}^{(1)}}\right) \cdot \ldots \cdot h_{k_{r}}\left(x_{s_{1}^{(r)}}, \ldots, x_{s_{k_{1}}^{(r)}}\right),
$$

где $\left\{s_{1}^{(i)}, \ldots, s_{k_{i}}^{(i)}\right\} \cap\left\{s_{1}^{(j)}, \ldots, s_{k_{j}}^{(j)}\right\}=\varnothing, \quad 1 \leq i<j \leq r$.

Пусть $r \geq 2$. Без ограничения общности будем считать, что $s_{1}^{(1)}=1$, $s_{2}^{(1)}=2, s_{1}^{(2)}=3, s_{2}^{(2)}=4$. Рассмотрим следующие выполняющие векторы функции $f$ :

$$
\alpha=(0,0,0,1,1, \ldots, 1), \beta=(0,1,0,0,1, \ldots, 1), \gamma=(1,1,1,1,1, \ldots, 1) .
$$

Вектор $\delta=v(\alpha, \beta, \gamma)=(0,1,0,1,1, \ldots 1)$ является, очевидно, выполняющим вектором функции $f$ и является производным вектором. Следовательно, наше допущение неверно, а значит, $r=1$.

В обратную сторону утверждение теоремы очевидно.

Следствие 2.4. В условиях теоремы 2.3 мощность системы образующих равна мощности множества выполняющих векторов.

ЗАМЕЧАНИЕ. Если функция $f$ существенно зависит от всех переменных и удовлетворяет условию теоремы 2.3 , то ее вес, а следовательно, и мощность системы образующих алгебры $E_{f}$ равны $n+1$. Следующая теорема устанавливает верхнюю оценку мощности минимальной системы образующих для всех функций из $\mathrm{WP} \cap \mathrm{WN}$, исходя из мощности множеств неразложимых и конеразложимых векторов.

Обозначим через $m_{f}$ минимальную мощность системы образующих множества выполняющих векторов биюнктивной функции $f$.

Теорема 2.5. Пусть $f\left(x_{1}, \ldots, x_{n}\right) \in \mathrm{WP} \cap \mathrm{WN}$, граф $G(f)(X)$ не имеет сильно связных компонент. Пусть $p$ - число начальных вершин в графе $G(f)(X)$, a $q$ - число конечных вериин этого графа. Если $p=1$ или $q=1$, то $m_{f} \leq n+1$. Если же $p>1$ и $q>1$, то $m_{f} \leq n+2$. 
Доказательство. Пусть $p=1$. Тогда $1_{n} \in R_{f}$ по утверждению 1.2, и очевидно, что $E_{f}=\left\langle R_{f}\right\rangle$.

Пусть $\quad p \neq 1, \quad$ но $\quad q=1 . \quad$ Если $\quad g\left(x_{1}, \ldots, x_{n}\right)=f\left(x_{1} \oplus 1, \ldots, x_{n} \oplus 1\right), \quad$ то

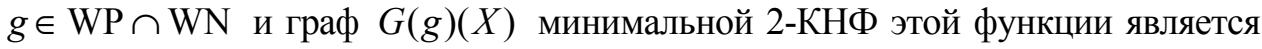
обратным к графу $G(f)(X)$. Тогда легко показать, что $E_{g}=E_{f} \oplus 1_{n}, 1_{n} \in R_{g}$, $R_{f} \oplus 1_{n}=$ со- $R_{g}, E_{g}=\left\langle R_{g}\right\rangle$. Следовательно, $E_{f}=\left\langle\right.$ со- $\left.R_{f}\right\rangle$, и мощность порождающего множества не превосходит $n+1$.

Если $p \neq 1, q \neq 1$, то, очевидно, $E_{f}=\left\langle R_{f} \cup\left\{1_{n}\right\}\right\rangle=\left\langle\right.$ со- $\left.R_{f} \cup\left\{0_{n}\right\}\right\rangle$.

Если теорема 2.5 дает оценку на мощность системы образующих любой функции из WP $\cap \mathrm{WN}$, то следующая теорема дает оценку минимальной мощности системы образующих путем построения примера функции с такой системой.

Теорема 2.6. Верно неравенство

$$
\min _{f \in \operatorname{Bi}(n)} m_{f} \leq\lceil\sqrt{2 n+1}\rceil .
$$

Доказательство. Для доказательства утверждения достаточно построить функцию $f\left(x_{1}, \ldots, x_{n}\right) \in \mathrm{WP} \cap \mathrm{WN}$, для которой $m_{f} \leq\lceil\sqrt{2 n+1}\rceil$. Для этого воспользуемся теоремой 1.4. Будем строить граф $G_{1}=G(f)(X)$ минимальной 2-КНФ без проходных вершин. Пусть $P=\left\{x_{1}, \ldots, x_{k}\right\}-$ множество начальных вершин графа, а $Q=\left\{x_{k+1}, \ldots, x_{n}\right\}-$ множество его конечных вершин. Проиндексируем элементы множества $Q$ неупорядоченными парами чисел $\{i, j\}: 1 \leq i<j \leq k$ и в каждую вершину из $Q$ вида $y_{i, j}$ проведем дуги из вершин $x_{i}$ и $x_{j}$. В результате мы получаем двудольный граф, не содержащий проходных вершин. При этом должно выполняться неравенство

$$
n-k=|Q| \leq\left(\begin{array}{l}
k \\
2
\end{array}\right)=\frac{k(k-1)}{2},
$$

T. e.

$$
k^{2}+k-2 n \geq 0 .
$$

Найдем минимальное целое значение $k=k(n)$, удовлетворяющее данному неравенству. Положительным корнем уравнения $k^{2}+k-2 n=0$ является значение

$$
k_{0}=\frac{\sqrt{8 n+1}}{2}-\frac{1}{2}<\sqrt{2 n+1} \leq\lceil\sqrt{2 n+1}\rceil .
$$

Поэтому $\min _{f \in B i(n)} m_{f} \leq\lceil\sqrt{2 n+1}\rceil$, что и требовалось доказать. 


\section{§ 3. Построение систем образующих на основе задачи о покрытии множеств}

Пусть $M \subset V_{n}$. Упорядоченный набор

$$
\lambda=\left(i_{1}, \ldots, i_{r}, a_{1}, \ldots, a_{r}\right), 1 \leq i_{1}<\ldots<i_{r} \leq n, a_{1}, \ldots, a_{r} \in\{0,1\}
$$

будем называть недопустилой $r$-грамлой множества $M$, если $M_{i_{1} \ldots i_{r}}^{a_{1} \ldots a_{r}}=\varnothing$. В частности, можно говорить о недопустимых биграммах, триграммах и т. д. Далее рассматривается случай $r=2$. Если $M_{i_{1} i_{2}}^{a_{1} a_{2}} \neq \varnothing$, будем говорить о допустимой биграмме.

Каждый вектор $\alpha \in V_{n}$, рассматриваемый как одноэлементное множество, имеет в точности $3 \cdot\left(\begin{array}{l}n \\ 2\end{array}\right)$ недопустимых и $\left(\begin{array}{l}n \\ 2\end{array}\right)$ допустимых биграмм. Обозначим множество недопустимых биграмм вектора $\alpha$ через $Z(\alpha)$. Очевидно, что $\bigcup_{\alpha \in V_{n}} Z(\alpha)=\Lambda$. По определению $|\Lambda|=4 \cdot\left(\begin{array}{l}n \\ 2\end{array}\right)$. Пусть $U(\alpha)-$ множество допустимых биграмм для $\alpha$, т. е. $U(\alpha)=\Lambda \backslash Z(\alpha)$. Ясно, что $|U(\alpha)|=\left(\begin{array}{l}n \\ 2\end{array}\right)$. Для булевой функции $f\left(x_{1}, \ldots, x_{n}\right)$ положим

$$
U(f)=\bigcup_{\alpha \in E_{f}} U(\alpha), \quad Z(f)=\bigcap_{\alpha \in E_{f}} Z(\alpha) .
$$

Легко видеть, что для любых булевых функций $f_{1}$ и $f_{2}$ выполнены равенства

$$
\begin{aligned}
& U\left(f_{1} \wedge f_{2}\right)=U\left(f_{1}\right) \cap U\left(f_{2}\right), \\
& U\left(f_{1} \vee f_{2}\right)=U\left(f_{1}\right) \cup U\left(f_{2}\right), \\
& Z\left(f_{1} \wedge f_{2}\right)=Z\left(f_{1}\right) \cup Z\left(f_{2}\right), \\
& Z\left(f_{1} \vee f_{2}\right)=Z\left(f_{1}\right) \cap Z\left(f_{2}\right) .
\end{aligned}
$$

Очевидно также, что если $f_{1} \leq f_{2}$, то

$$
U\left(f_{1}\right) \subset U\left(f_{2}\right), \quad Z\left(f_{2}\right) \subset Z\left(f_{1}\right) .
$$

Следующая теорема является вариантом теоремы, доказанной в [1]. 
Булеву биюнктивную функцию $f$ назовем наилучшим верхним биюнктивным аналогом функции $g$, если $E_{g} \subseteq E_{f}$ и для любой биюнктивной функции $f^{\prime}$ из условия $E_{g} \subseteq E_{f}$, следует, что $E_{f} \subseteq E_{f}$. Методы построения наилучших верхних биюнктивных аналогов обсуждались в статье [5].

Теорема 3.1. Булева биюнктивная функция $f$ является наилучшим верхним биюнктивным аналогом функции $g$ тогда и только тогда, когда $U(f)=U(g), \quad Z(f)=Z(g)$.

Доказательство. В силу теоремы 3 из [1] биюнктивная функция $f$ является наилучшим верхним биюнктивным аналогом функции $g$ тогда и только тогда, когда $\left\langle E_{g}\right\rangle=E_{f}$. Пусть $f$ - наилучший верхний биюнктивный аналог $g$. Тогда $g \leq f$ и $Z(f) \subset Z(g)$. Докажем обратное включение. Предположим, что $\lambda=(i, j, c, d) \in Z(g) \backslash Z(f)$. Это означает, что $g_{i j}^{c \oplus 1} d \oplus 1 \equiv 0$, но найдется

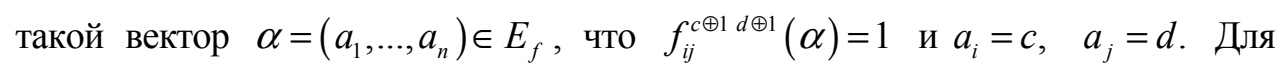
удобства дальнейших рассуждений будем считать, что $c=d=0, \quad i=1, \quad j=2$.

Рассмотрим последовательность множеств $N_{0}=E_{f}, \quad N_{m+1}=\left\langle N_{m}\right\rangle_{1}$, $m \geq 0$. Так как $\left\langle E_{g}\right\rangle=E_{f}, \alpha \in E_{f}$, то существует такое $k$, что множество $N_{k-1}$ не содержит векторов, у которых первые 2 координаты равны 0 , а множество $N_{k}$ содержит такие векторы. Пусть первые координаты вектора $\alpha$ равны 0 . Тогда в $N_{k-1}$ найдутся такие векторы $\beta, \gamma, \delta$, что $\alpha=v(\beta, \gamma, \delta)$. Положим $\beta=\left(b_{1}, b_{2}, \ldots\right), \quad \gamma=\left(y_{1}, y_{2}, \ldots\right), \quad \delta=\left(z_{1}, z_{2}, \ldots\right)$. Тогда выполняется система булевых равенств:

$$
\left\{\begin{array}{l}
b_{1} \vee b_{2}=1, \\
y_{1} \vee y_{2}=1, \\
z_{1} \vee z_{2}=1, \\
b_{1} y_{1} \vee b_{1} z_{1} \vee y_{1} z_{1}=0, \\
b_{2} y_{2} \vee b_{2} z_{2} \vee y_{2} z_{2}=0 .
\end{array}\right.
$$

Непосредственной проверкой легко убедиться, что система (3.3) несовместна. Следовательно, исходное предположение неверно и $Z(f)=Z(g)$.

Пусть теперь $Z(f)=Z(g)$ и $f-$ биюнктивная функция. Покажем, что $\left\langle E_{g}\right\rangle=E_{f}$. Пусть $h-$ такая биюнктивная функция, что $E_{h}=\left\langle E_{g}\right\rangle$. Тогда $h-$ наилучший верхний биюнктивный аналог $g$, и $Z(h)=Z(g)=Z(f)$ в силу доказательства первой части теоремы. Докажем, что $h \equiv f$. 
В самом деле, $\lambda=(i, j, a, b) \in Z(h)$ тогда и только тогда, когда функция $\psi=x_{i}^{a} \vee x_{j}^{b}-$ имплицента $h$. Тогда $\psi-$ имплицента $f$. Аналогично, верно обратное. Следовательно, биюнктивные функции $f$ и $h$ имеют одинаковые наборы имплицент. Значит, $h \equiv f$.

Следствие 3.2. Пусть $M \subset V_{n}, N=\langle M\rangle$. Тогда $U(M)=U(N)$.

Доказательство. Для доказательства следствия достаточно положить $f=f_{M}, g=f_{N}$ и применить теорему 3.1.

С помощью теоремы 3.1 легко получается следующее утверждение.

Теорема 3.3. Пусть $f\left(x_{1}, \ldots, x_{n}\right)$ - биюнктивная функиия. Для множества векторов $M \subset E_{f}$ условие $\langle M\rangle=E_{f}$ выполняется тогда и только тогда, когда

$$
U(f)=\bigcup_{\alpha \in M} U(\alpha)
$$

Доказательство. Если $M \subset E_{f}$, то $\langle M\rangle=E_{f}$ тогда и только тогда, когда $f$ - такой наилучший верхний аналог функции $g$, что $E_{g}=M$. В силу (3.1) $U(g)=\bigcup_{\alpha \in M} U(\alpha)$. Для завершения доказательства достаточно воспользоваться теоремой 3.1.

Фактически теорема 3.3 говорит о том, что множество векторов М является системой образующих алгебры $E_{f}<\Omega_{n}$ тогда и только тогда, когда множества $U(\alpha)$ образуют покрытие множества $U(f)$. Естественно возникает задача построения минимального покрытия - минимальной системы образующих.

Если биюнктивная функция $f$ задана сокращенной 2-КНФ вида

$$
f\left(x_{1}, \ldots, x_{n}\right)=\sum_{i=1}^{t}\left(x_{s_{i 1}}^{a_{i 1}} \vee x_{s_{i 2}}^{a_{i 2}}\right),
$$

то положим $\lambda_{i}=\left(s_{i 1}, s_{i 2}, a_{i 1} \oplus 1, a_{i 2} \oplus 1\right), i=1, \ldots, t$. Ясно, что при этом $Z(f)=\left\{\lambda_{1}, \ldots, \lambda_{t}\right\}$. Тогда $U(f)=\Lambda \backslash Z(f)$. По 2-КНФ (3.4) легко получить множество $U(f)$. Ясно, что если $\lambda=(i, j, c, d) \in U(f)$, то $\left\|f_{i j}^{c d}\right\| \geq 1$. Кроме того, поскольку функция $f_{i j}^{c d}-$ биюнктивная, можно с линейной сложностью от длины ее 2-КНФ найти выполняющий вектор $\alpha^{\prime}$ и по нему построить такой вектор $\alpha=\left(a_{1}, \ldots, a_{n}\right) \in E_{f}$, что $a_{i}=c, a_{j}=d$. Проведя подобные построения 
для всех $\lambda \in U(f)$, построим систему образующих для алгебры $E_{f}$. Проведенные рассуждения доказывают следующее утверждение.

Утверждение 3.4. Задача построения системь образующих $M$ для подалгебры $E_{f}$ для любой 2-КНФ $f$ вида (0.1) имеет сложность не более $O\left(n^{4}\right)$, мощность множества М не превосходит $n(n-1)$.

Обратимся теперь к теореме 1.1. Если $f \in \mathrm{WP}$, то мы можем представить функцию $f$ в виде произведения $f=g \cdot h$, где $g \in \mathrm{Bi} \cap \mathrm{WP}, \quad h \in \mathrm{WP}$ и функция $h$ не имеет имплицент длины, меньшей 3 . Пусть, далее, $R_{f}-$ множество неразложимых векторов функции $f$. Положим также $U(M)=$ $=U\left(f_{M}\right), \quad Z(M)=Z\left(f_{M}\right)$ для произвольного множества $M \subset V_{n}$.

Теорема 3.5. Во введенных выше условиях верны равенства

$$
U\left(R_{f} \cup\left\{1_{n}\right\}\right)=U(g), \quad Z\left(R_{f} \cup\left\{1_{n}\right\}\right)=Z(g) .
$$

В частности, функиия $f$ не имеет имплищент длины, меньшей 3, тогда и только тогда, когда $U\left(R_{f} \cup\left\{1_{n}\right\}\right)=\Lambda, \quad Z\left(R_{f} \cup\left\{1_{n}\right\}\right)=\varnothing$.

Доказательство теоремы следует из следствия 3.2.

Лемма 3.6. Длина сокращенной 2-КНФ биюнктивной функции от $n$ neременных не превосходит величины $n(n-1)$.

Доказательство. Пусть $f$ задана формулой (3.4). Для каждой из $\left(\begin{array}{l}n \\ 2\end{array}\right)$ пар $i, j$ таких, что $1 \leq i<j \leq n$, в (3.4) подсчитаем число $k_{i j}$ элементарных дизъюнкций от переменных $x_{i}$ и $x_{j}$. Очевидно, что $0 \leq k_{i j} \leq 2$. В противном случае функция $f$ имеет имплиценты длины 1. Следовательно, $t=\sum_{1 \leq i<j \leq n} k_{i j} \leq$ $\leq 2\left(\begin{array}{l}n \\ 2\end{array}\right)=n(n-1)$. С другой стороны, данная оценка достижима на функции

$$
f\left(x_{1}, \ldots, x_{n}\right)=\underset{1 \leq i<j \leq n}{\&}\left(\left(x_{i} \vee \bar{x}_{j}\right)\left(\bar{x}_{i} \vee x_{j}\right)\right)=\underset{1 \leq i<j \leq n}{\&}\left(x_{i} \oplus x_{j} \oplus 1\right) .
$$

Для получения верхних оценок мощности систем образующих воспользуемся следующим подходом. Пусть функция $f$ биюнктивна и представлена в виде сокращенной 2-КНФ (3.4). Рассмотрим множества $U(f)$ и $Z(f)$. Пусть выполнено условие: если $\lambda=(i, j, c, d) \in U(f)$, то $\left\|f_{i j}^{c d}\right\| \geq m>1$, где $m-$ параметр. 
Теорема 3.7. В приведенных условиях существует система образующих алгебры $E_{f}$, содержамая не более

$$
\left\lceil\frac{\|f\|}{m} \ln \left(\frac{2 m n(n-1)}{\|f\|}\right)\right\rceil+\frac{\|f\|}{m}
$$

векторов.

Доказательство. Построим $(0,1)$-матрицу $A$ по следующему правилу. В матрице будет $p=\|f\|$ строк и $s=|U(f)|$ столбцов. В соответствии с леммой 3.6 для величины $s$ верна оценка

$$
n(n-1) \leq s \leq 2 n(n-1) .
$$

Строки матрицы $A$ проиндексированы векторами из $E_{f}$, а столбцы элементами $\lambda \in U(f)$. Для каждого $\alpha \in E_{f}$ в соответствующей строке матрицы $A$ поставим единицы на местах, соответствующих комбинациям $\lambda \in U(\alpha)$. На остальных местах поставим нули. В итоге, матрица $A$ обладает следующими свойствами:

- вес каждой строки равен $\left(\begin{array}{l}n \\ 2\end{array}\right)$;

- вес каждого столбца не меньше $m$;

- число столбцов $T \geq 3 / 2 \cdot n(n-1)$ (по лемме 3.6 );

- число строк равно $\|f\|$.

В такой трактовке задача построения минимальной по мощности системы образующих преобразуется в задачу построения минимального покрытия матрицы $A$ строками. Для получения оценок воспользуемся подходом, изложенным в [7] и [8]. В основу положим использование градиентного («жадного») алгоритма. На каждом шаге алгоритма будем выбирать строку, покрывающую наибольшее из оставшихся количество столбцов. Если таких строк несколько, то берем любую из них.

Предположим, что для получения покрытия алгоритму необходимо сделать $q$ шагов. Пусть на первых $k$ шагах $(k \in 1, \ldots, q)$ были выбраны строки с номерами $i_{1}, \ldots, i_{k}$. Пусть, далее, $A_{k}$ - матрица, полученная из $A$ путем удаления этих строк и покрываемых ими столбцов. Данная матрица будет иметь $p_{k}=p-k$ строк и $s_{k}$ столбцов. Будем считать, что $p_{0}=p, s_{0}=s$.

Так как на каждом шаге алгоритма добавляется покрытие хотя бы одного столбца, то

$$
q \leq k+s_{k} .
$$


Кроме того, в каждом столбце матрицы $A_{k}$ будет не менее $m$ единиц, поскольку в эту матрицу входят еще не покрытые столбцы. Тогда общее число единиц в матрице $A_{k}$ не меньше $m s_{k}$. Выбирая на $(k+1)$-м шаге строку максимального веса, получаем, что в ней должно быть не менее $m s_{k} / p_{k}$ единиц. Значит, эта строка покроет не менее $m s_{k} / p_{k}$ столбцов, и

$$
s_{k+1} \leq s_{k}-\frac{m s_{k}}{p_{k}}=s_{k}\left(1-\frac{m}{p_{k}}\right) .
$$

Положим $\gamma=m / p, \delta_{k}=s_{k} / s$. Тогда полученное неравенство можно переписать в виде

$$
\delta_{k+1} \leq \delta_{k}\left(1-\frac{\gamma p}{p-k}\right) .
$$

Поскольку $\delta_{0}=1$, по индукции находим, что

$$
\delta_{k} \leq \prod_{i=0}^{k-1}\left(1-\frac{\gamma p}{p-i}\right) \leq(1-\gamma)^{k} \leq e^{-\gamma k}, \quad k=0, \ldots, q .
$$

Тогда, с учетом неравенства $q \leq k+s_{k}$,

$$
q \leq k+s e^{-\gamma k}, k=0, \ldots, q .
$$

Функция $k+s e^{-\gamma k}$ достигает минимума при $k=\frac{1}{\gamma} \ln \left(\gamma_{s}\right)$. Подставив значение $k=\left\lceil\frac{1}{\gamma} \ln \left(\gamma_{s}\right)\right\rceil$, получим неравенство

$$
q \leq\left\lceil\frac{1}{\gamma} \ln \left(\gamma_{S}\right)\right]+\frac{1}{\gamma} .
$$

Воспользовавшись условиями $s \leq 2 n(n-1), \gamma=\frac{m}{p}$, получим

$$
q \leq\left\lceil\frac{p}{m} \ln \left(\frac{m s}{p}\right)\right\rceil+\frac{p}{m} \leq\left\lceil\frac{p}{m} \ln \left(\frac{2 m n(n-1)}{p}\right)\right\rceil+\frac{p}{m},
$$

что и требовалось доказать.

Непосредственно из доказанных теорем вытекает метод построения систем образующих для множеств выполняющих векторов биюнктивных функций, основанный на задаче о покрытии множеств. С целью проверки эффективности этого метода М. А. Киввой были построены системы образующих $M_{f}$ множеств выполняющих векторов биюнктивных функций $f$ от 3, 4 и 5 переменных. Построение покрытий проводилось с помощью градиентного алгоритма. После построения указанных систем были подсчитаны $E_{1}-$ средняя 
мощность системы образующих и $E_{2}-$ среднее отношение мощности полученной системы образующих к мощности всего множества выполняющих векторов:

$$
E_{1}=\frac{1}{|\operatorname{Bi}(n)|} \sum_{[f]}|[f]|\left|M_{f}\right|, \quad E_{2}=\frac{1}{|\operatorname{Bi}(n)|} \sum_{[f]}|[f]| \frac{\left|M_{f}\right|}{\|f\|} .
$$

Полученные результаты приведены в таблице 3.1.

Таблица 3.1.

\begin{tabular}{|c|c|c|}
\hline$n$ & $E_{1}$ & $E_{2}$ \\
\hline 3 & 2,77 & 0,8 \\
\hline 4 & 4,56 & 0,86 \\
\hline 5 & 5,46 & 0,6 \\
\hline
\end{tabular}

\section{Список литературы}

1. Тарасов A. B. Универсальные алгебры, порождаемые множествами выполняющих векторов биюнктивных и $r$-юнктивных булевых функций // Математические вопросы криптографии. - 2011. - Т. 2. Вып. 3. - С. 75-98.

2. Aspvall B., Plass M. F., Tarjan R. E. A linear-time algorithm for testing the truth of certain quantified Boolean formulas // Inform. Process. Lett. — 1979. V. 8. - P. 121-123.

3. Тарасов А. В. О свойствах функций, представимых в виде 2-КНФ// Дискретная математика. - 2001. - Т. 13. Вып. 4. - С. 99-115.

4. Горшков С. П. Применение теории NР-полных задач для оценки сложности решения систем булевых уравнений // Обозр. прикл. и промышл. матем. М.: ТВП, 1995. - Т. 2. Вып. 3. - С. 325-398.

5. Горшков С. П. О сложности распознавания мультиаффинности, биюнктивности, слабой положительности и слабой отрицательности булевых функций // Обозр. прикл. и промышл. матем. - М.: ТВП, 1997. - Т. 4. Вып. 2. - С. 216-237.

6. Горшков С. П. О пересечении классов мультиаффинных, биюнктивных, слабо положительных и слабо отрицательных булевых функций // Обозр. прикл. и промышл. матем. - М.: ТВП, 1997. - Т. 4. Вып. 2. - С. 238-259.

7. Райгородский $A$. Системы общих представителей и их приложения в геометрии. - М.: МЦНМО, 2006.

8. Сачков В. Н., Тараканов В. Е. Комбинаторика неотрицательных матриц. М.: ТВП, 2000. - $448 \mathrm{c}$.

9. Гретиер Г. Общая теория решеток. - М.: Мир, 1982. — 452 c. 